\title{
Functional roles of protein phosphatase 4 in multiple aspects of cellular physiology: a friend and a foe
}

\author{
Jaehong Park ${ }^{1} \mathcal{E}$ Dong-Hyun Lee ${ }^{2,3, *}$ \\ ${ }^{1}$ School of Biological Sciences and Biotechnology Graduate School, Chonnam National University, Gwangju 61186, ${ }^{2}$ Department of \\ Biological Sciences, College of Natural Sciences, Chonnam National University, Gwangju 61186, ${ }^{3}$ Research Center of Ecomimetics, \\ Chonnam National University, Gwangju 61186, Korea
}

Protein phosphatase 4 (PP4), one of serine/threonine phosphatases, is involved in many critical cellular pathways, including DNA damage response (DNA repair, cell cycle regulation, and apoptosis), tumorigenesis, cell migration, immune response, stem cell development, glucose metabolism, and diabetes. PP4 has been steadily studied over the past decade about wide spectrum of physiological activities in cells. Given the many vital functions in cells, PP4 has great potential to develop into the finding of key working mechanisms and effective treatments for related diseases such as cancer and diabetes. In this review, we provide an overview of the cellular and molecular mechanisms by which PP4 impacts and also discuss the functional significance of it in cell health. [BMB Reports 2020; 53(4): 181-190]

\section{INTRODUCTION}

A number of cellular pathways are regulated through the reversible phosphorylation of proteins orchestrated by kinases and phosphatases, a central mechanism in the regulation of signal transduction (1). Protein phosphatases can be classified into four gene families each with distinct functions in cells: (i) Serine/threonine phospho-protein phosphatase (PPP); (ii) $\mathrm{Mg}^{2+}$ dependent protein phosphatase (PPM/PP2C); (iii) Phosphotyrosine phosphatase (PTP); (iv) Asp-based protein phosphatase (1-4). PPPs are the most highly conserved phosphatases in eukaryotes, and more than $80 \%$ phosphatase activity is contributed to this family. PPPs are further divided into seven subgroups (PP1, PP2A, PP2B, PP4, PP5, PP6, and PP7) based on sequence, structure, and biochemical properties. Approximately 40 catalytic subunits derived from PPPs and PPM/PP2C are responsible for dephosphorylating the majority of Ser/Thr phosphoresidues on proteins, whereas more than 400 kinases

*Corresponding author. Tel: +82-62-530-3418; Fax: +82-62-5303409; E-mail: donghyunlee73@jnu.ac.kr

https://doi.org/10.5483/BMBRep.2020.53.4.019

Received 8 January 2020

Keywords: Genomic stability, Glucose homeostasis, Immunity, Neuronal development, Protein phosphatase 4 are in charge of phosphorylation of Ser/Thr residues. This implies that these phosphatases should be highly regulated, and the majority of catalytic activities is usually attributed to a wide variety of regulatory subunits, forming functionally unique complexes with a limited number of catalytic subunits (Fig. 1) (1-3).

Protein phosphatase 4 (PP4) complex is comprised of one catalytic subunit (PP4C) and five regulatory subunits (PP4R1, PP4R2, PP4R3 $\alpha$, PP4R3 $\beta$, and PP4R4), typically forming the functional heterodimer (PP4C/PP4R1 or PP4C/PP4R4) or heterotrimer (PP4C/PP4R2/PP4R3 $\alpha$ or PP4C/PP4R2/PP4R3 $\beta$ ) in cells (Fig. 2) (3-10). PP4C, a catalytic subunit of PP4, was the first identified in 1993 as PPX (designated as PPP4 in human genome nomenclature) and indicated $65 \%$ identical to PP2AC $\alpha$ and PP2AC $\beta$. Given the $<70 \%$ identity to other phosphatases including PP1, PP2A and PP2B, PP4C cannot be considered as isoforms of these proteins (4). Also, it did not show physical interaction with other phosphatases tested and had the distinct functions $(1,4)$. The regulatory subunits of PP4 are highly conserved across mammals, yeast and plants. The heterotrimer complex, PP4C-PP4R2-PP4R3 (PPH3-YBL1046W-PSY2), sharing a high homology between human and $S$. cerevisiae, functions in DNA damage response (DDR) in the both organisms (9, 11-13). Arabidopsis also contains regulatory subunits of PP4, PP4R2 domain (putative homologue of PP4R2 in human), and SMEK-1 domain (homologue of PP4R3 in human), even though their functional properties are largely undiscovered. In the beginning, the main effort of PP4 research was focused on the DDR, thus finding that phosphatase directly dephosphorylates many essential proteins particularly in DDR, facilitating efficient DNA repair and check point control: RPA2 (replication protein A2), KAP-1 (KRAB-associated protein-1), $\gamma-\mathrm{H} 2 \mathrm{AX}$ (gamma $\mathrm{H} 2 \mathrm{~A}$ histone family member $\mathrm{X}$ ), 53BP1 (p53 binding protein 1), DBC1 (deleted in breast cancer-1) and Rad53 (5-9, 14). However, the results of more than a decade of studies in various fields of cell physiology showed that PP4 had many other essential physiological activities as well as DDR: genomic stability (cancer, cell cycle control, and DNA repair), immune response, glucose homeostasis, and neuron development. 


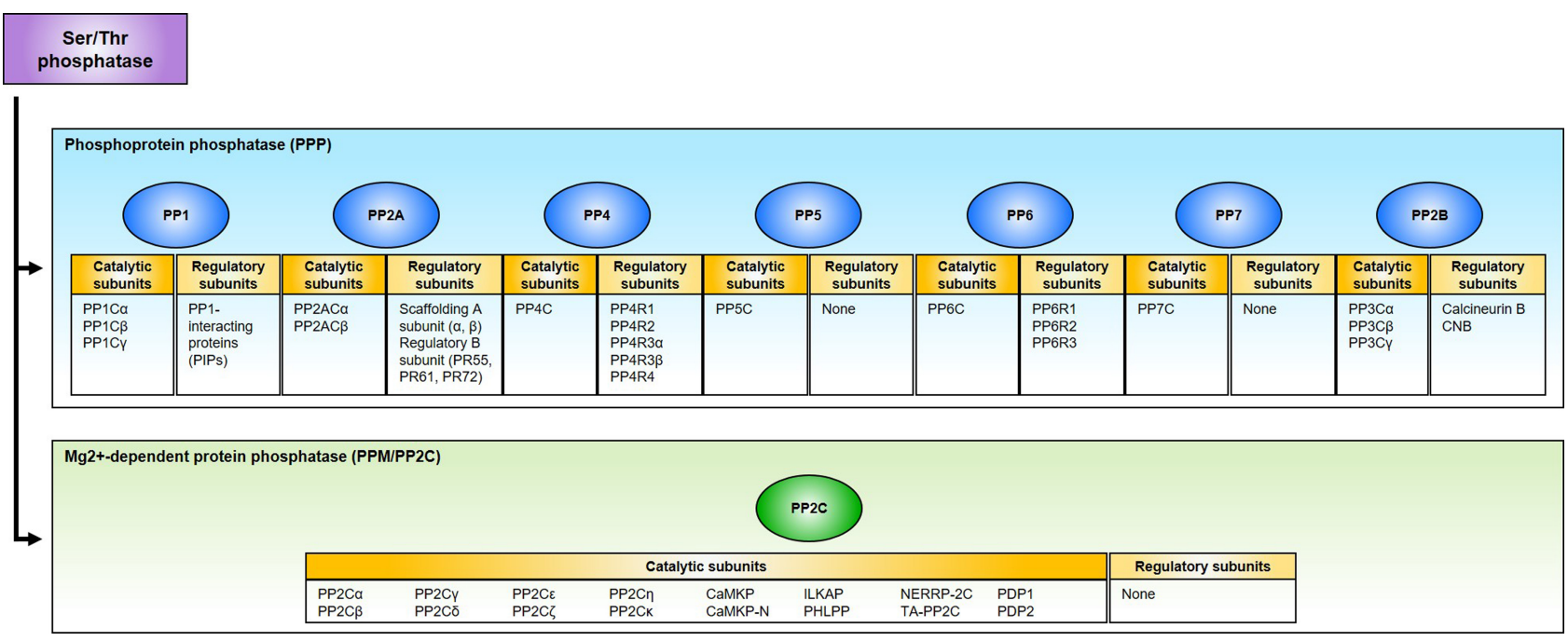

Fig. 1. Classification of Ser/Thr phosphatase. Ser/Thr phosphatases are classified into phosphoprotein phosphatases (PPP) and $\mathrm{Mg}^{2+}-$ dependent protein phosphatase (PPM/PP2C). The PPP family can be further divided into seven subgroups: PP1, PP2A, PP2B, PP4, PP5, PP6, and PP7. Most Ser/Thr phosphatases are in the complex with their catalytic and regulatory subunits, except for PP5, PP7, and PP2C, which are solely comprised of catalytic subunits.

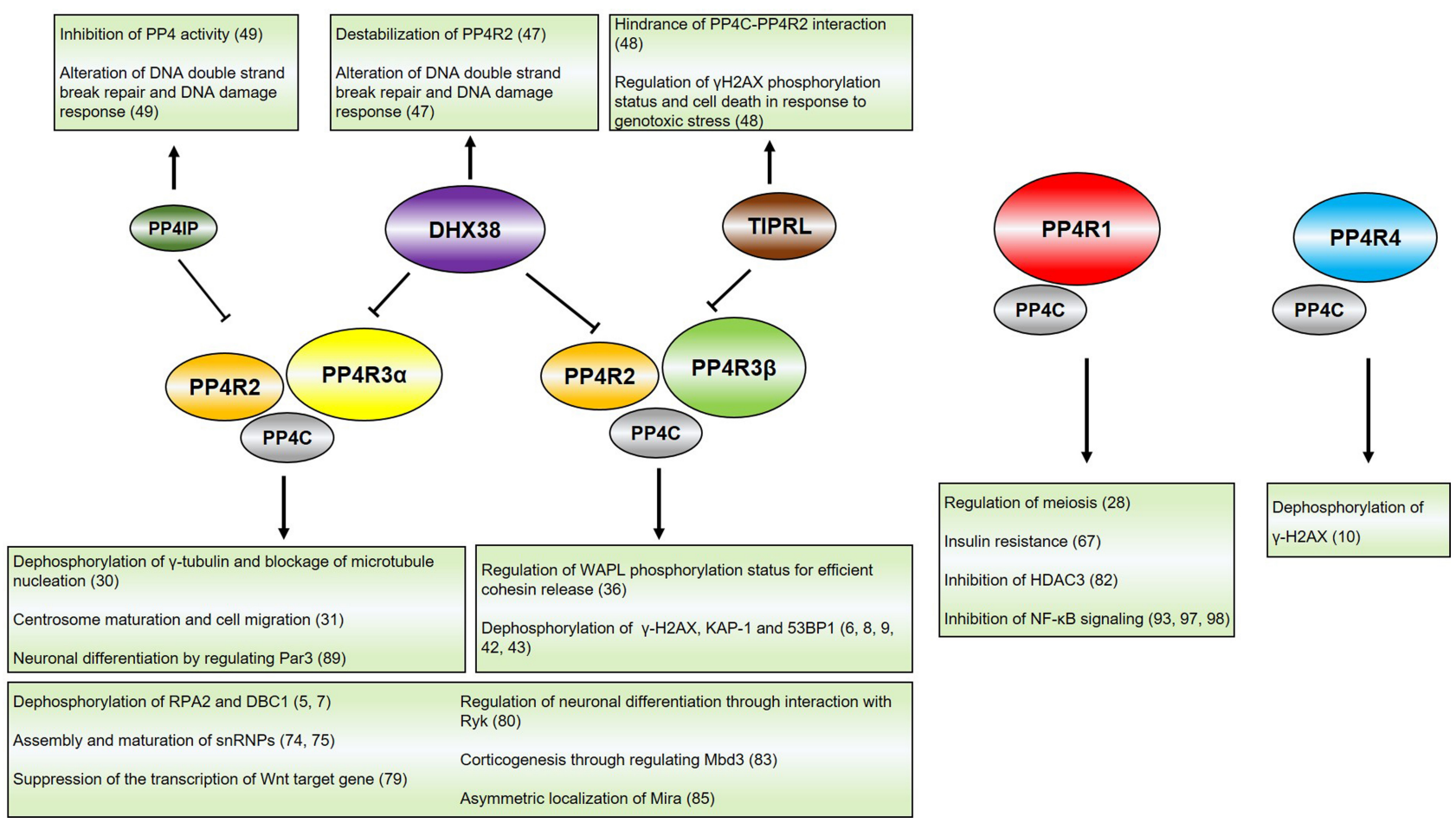

Fig. 2. Protein phosphatase 4 complex and its inhibitory proteins. PP4 complex is comprised of its catalytic subunit (PP4C) and regulatory subunits (PP4R1, PP4R2, PP4R3 $\alpha$, PP4R3 $\beta$, and PP4R4). PP4C interacts with PP4R1 or PP4R4 as a heterodimer, and PP4R2 and PP4R3 $\alpha / \beta$ as a heterotrimer. Target substrates of PP4 complex to be dephosphorylated are determined via regulatory subunits. The activity of PP4 complexes can be regulated by the inhibitory proteins, including PP4IP, DHX38, and TIPRL. While PP4C/PP4R2/PP4R3 $\alpha$ complex is regulated by PP4IP or DHX38, PP4C/PP4R2/PP4R3 $\beta$ complex is regulated by TIPRL or DHX38. Inhibitory proteins for PP4C/PP4R1 or PP4C/ PP4R4 complex have not been elucidated. The reference numbers for the information are indicated. PP4IP, PP4 inhibitory protein; DHX38, DEAH box polypeptide 38; TIPRL, Tip41-like protein. 


\section{THE ROLES OF PP4 IN VARIOUS CELLULAR PHYSIOLOGY}

In this review, we will summarize the recent understanding and the biological significance of the roles of PP4 in a wide range of cellular physiology including genomic stability, immune response, glucose homeostasis, neuronal development, and plant physiology (Fig. 3).

\section{Genomic stability}

Genome is consistently exposed to DNA damages caused by endogenous or exogenous events. If genomic aberration occurs, cells will suffer from disasters such as cell death and transformation. Thus, it is necessary to maintain genomic integrity.

Cancer: It has been known that PP4 is overexpressed in human breast and lung tumors, stage II pancreatic ductal adenocarcinoma, colorectal carcinoma, and glioma (15-18), and also significantly increases JNK-1 (c-Jun N-terminal kinase 1) activity in prostate cancer cell lines, PC-3 and LNCaP, which is essential for proliferation and drug resistance (19), suggesting that PP4 could have oncogenic activity. However, in conflicting studies, PP4R2 gene is deleted in patients with acute myeloid leukemia (AML), required for proper DNA repair and functions as tumor suppressor protein (20). And PP4 recovers the expression of HPK1 (hematopoietic progenitor kinase 1), lost in more than $95 \%$ of pancreatic cancer through proteasome-mediated degradation, thus inhibiting cell proliferation of pancreatic cancer cells (21). Paradoxically, PP4 is reported to improve cell proliferation and block apoptosis through downregulation of DR4 (death receptor 4) and dephosphorylation of DBC1, leading to SIRT1 (NAD-dependent deacetylase sirtuin-1) derepression and, subsequently, blocking p53-dependent apoptosis (7, 18, 22, 23) in one respect, but in the other respect, it could impair proliferation and cause apoptosis by inducing low phosphorylation level of PEA-15 (phosphoprotein enriched in astrocytes of $15 \mathrm{kDa}$ ) and Bad (Bcl2 associated agonist of cell death) $(24,25)$. Thus, further studies are required to understand the discrepancy in PP4

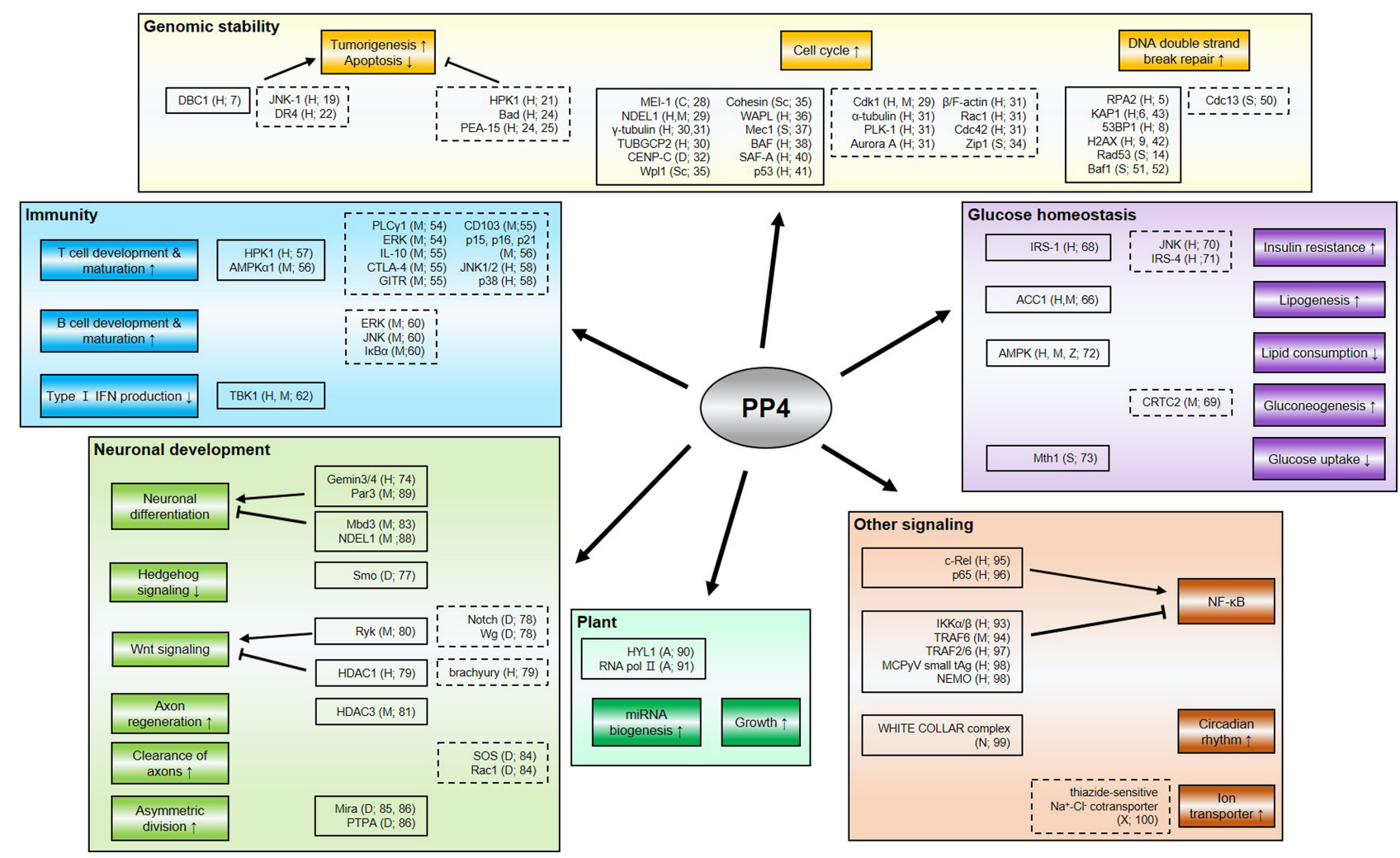

Fig. 3. Multiple roles of protein phosphatase 4 in various cellular physiology. This schematic depicts the regions wherein PP4 participates and the genes regulated by or associated with PP4. The proteins in the closed boxes directly interact with PP4, but not all of them are targets to be dephosphorylated, whereas the proteins in the dashed boxes are indirectly regulated by PP4 or closely associated with the status of its expression. The organisms wherein the proteins are found and reference numbers are indicated; A, Arabidopsis thaliana; C, Caenorhabditis elegans; D, Drosophila melanogaster; H, human; M, mouse; N, Neurospora crassa; S, Saccharomyces cerevisiae; Sc, Schizosaccharomyces pombe; X, Xenopus laevis; Z, zebrafish. 
functions regarding tumor formation.

Cell cycle: For successful cell division, many processes should occur properly, such as microtubule organization and chromosome segregation. In various species, including human, PP4 has critical functions in microtubule organization (26-30). Caenorhabditis elegans has two pp4 catalytic subunit genes, pph-4.1 and pph-4.2, and PPH-4.1, not PPH-4.2, is necessary for the activation of microtubule nucleation (27). PPH-4.1 interacts with and activates MEI-1 (meiotic spindle formation protein 1), which must be inactivated via ubiquitindependent degradation after the meiosis to allow for the formation of the mitotic spindle, and also promotes the activity of PPFR-1 (homologue of PP4R1 in C. elegans), which regulates MEl-1 during mitosis (28). Also, abnormal phosphorylation of NDEL1 (nuclear distribution protein nudE-like 1) and uncontrolled activation of Cdk1 (cyclin-dependent kinase 1) by PP4C disruption induce excessive recruitment of Katanin p60 to the centrosome and consequently, cause microtubule defects (29). Also, it was reported that PP4C depletion leads aberrant regulation of $\alpha$-tubulin, $\gamma$-tubulin, PLK-1 (polo-like kinase 1), and Aurora A, and precludes the organization of actin cytoskeleton, possibly by inactivated Rho GTPases, Rac1 (Ras-related C3 botulinum toxin substrate 1) and Cdc42 (cell division control protein 42), thus impairing centrosome maturation and cell migration (31). In addition, PP4C interacts with $\gamma$-tubulin and TUBGCP2 (tubulin gamma complex associated protein 2) and dephosphorylates $\gamma$-tubulin (30). When the cells are arrested in mitosis with spindle toxins such as nocodazole or paclitaxel, PP4 activity is inhibited through phosphorylation of its regulatory subunit PP4R2 and PP4R3 $\alpha$, thus leading the blockage of microtubule nucleation by $\gamma$-tubulin. Kinetochore assembly is also required for PP4-CENP-C (centromeric protein C) interaction in Drosophila (32). Additionally, PP4 is related to chromosome pairing and crossover formation (33). Pph3A mutants have defects in crossover repair and centromere pairing, with upregulated phosphorylation of Zip1 (34). And also, PP4 regulates anti-cohesin pathway through interaction with Wpl1, cohesin and WAPL $(35,36)$. Furthermore, Pph3, concerted with but independent to Slx4, is required for termination of replicative stress signaling (37) and regulates checkpoint signaling by interaction with Mec1 (ATR yeast homolog) (38). Besides, knockdown of PP4C and PP4R2 increases the phosphorylation of BAF (barrier-to-autointegration factor) at Ser-4 site, which is important for normal nuclear envelop formation and cell cycle progression (39). However, overexpression of PP4, as well as the absence of PP4, also impairs proper cell cycle progression, but each condition results in different phenotypes; prometaphase arrest and interphase arrest, respectively (40). In prometaphase/metaphase transition, the interplay between PP4 and SAF-A (scaffold attachment factor A) is necessary (40).

Most studies for cell cycle regulation-related PP4 functions have focused on the mitosis and meiosis events. However, considering that PP4 is required for recovery from a check- point-induced arrest by dephosphorylating and inactivating p53 in G1 phase (41), it is feasible that PP4 could have essential functions in other cell cycle phases.

DNA repair: To prevent detrimental accidents by DNA damage, eukaryotes have evolved highly conserved and refined mechanisms, known as DNA damage response (DDR). DNA double strand break (DSB) is one of the most hazardous type of DNA lesions, restored mainly by non-homologous end joining (NHEJ) or homologous recombination (HR). To progress proper DNA damage response, timely dephosphorylation is required for counteracting the phosphorylation by kinases and in fact, PP4 has indispensable roles in these sophisticated processes (3)

Phosphorylated form of histone $\mathrm{H} 2 \mathrm{AX}$, named as $\gamma-\mathrm{H} 2 \mathrm{AX}$, is induced upon DNA damage and thus, regarded as the DNA damage marker. Aberration of dephosphorylation of $\gamma-\mathrm{H} 2 \mathrm{AX}$ by PP4 on time clearly impairs proper DDR and causes genomic instability $(9,42)$. PP4 also dephosphorylates RPA2, whose phosphorylation status is essential for DNA synthesis after DNA damage, G2/M phase checkpoint, and HR (5). Also, phosphorylation of 53BP1, one of most critical proteins in genomic stability and tumorigenesis, is mainly regulated by PP4 (8). 53BP1 must be highly phosphorylated at T1609/S1618 during mitosis so as not to be activated and thus, block error-prone NHEJ repair. However, PP4-mediated dephosphorylation of 53BP1 promotes the recruitment of 53BP1 to the DNA double strand break sites at the onset of G1 phase to secure genomic stability, but not in mitosis (8). When phospho-null mutants of 53BP1 (T1609A/S1618A) are recruited to DNA lesions in mitosis, chromosome segregation is largely impaired. Besides, DNA damage sites should be accessible to facilitate recruitment of DNA repair factors. When DNA lesions are generated, KAP-1, a transcriptional corepressor, is rapidly phosphorylated by ATM kinase, inducing relaxation of DNA lesions and thus promoting transcription of stressinduced genes, such as p21 and Gadd $45 \alpha$ (growth arrest and DNA damage-inducible alpha) to initiate DNA repair in heterochromatin. Right after DNA repair is completed, PP4 induces KAP-1 dephosphorylation to recover its corepressive function (6). PP4 is also involved in NHEJ pathway, possibly through altering KAP-1 phosphorylation (43). According to phosphoproteomic analysis (6), PP4 could participate in other DNA repair pathways, such as DNA mismatch repair and base excision repair in addition to DNA DSB repairs, implying that PP4 could be universal regulator for DNA repair.

Considering the critical roles of PP4 as a phosphatase, PP4 should be tightly regulated for efficient DDR. In fact, the post-translational modifications of PP4C and its regulatory subunits have significant impact on PP4 activity. PP4C methylation on C-terminal leucine site (Leu307) by LCMT-1 (leucine carboxyl methyltransferase 1) is required for stable PP4 complex, enzymatic activity to its targets, such as KAP-1 and 53BP1, and thus, DNA DSB repairs (HR and NHEJ) $(44,45)$. Also, PP4 regulatory subunits, like PP4R2, PP4R3 $\alpha$, and 
PP4R3 $\beta$, are phosphorylated to be inactivated in mitosis (30, 46). In particular, PP4R3 $\beta$ phosphorylation is required for maintaining 53BP1 phosphorylation and blocking premature 53BP1 foci formation in mitosis (45).

Recently, some endogenous PP4 inhibitors, such as DHX38 (DEAH box polypeptide 38), TIPRL (Tip41-like protein), and PP4IP (protein phosphatase 4 inhibitory protein), have been reported (47-49). DHX38 specifically interacts with PP4C in constitutive and damage-independent manner, and inhibits PP4 activity in vitro and in vivo, thus impacting DNA DSB repairs, and DNA synthesis after damage (47). PP4IP also interacts with PP4, but its inhibitory function depends on DNA damage and cell cycle, especially mitosis. PP4IP knockout promotes the stability of PP4C-PP4R2-PP4R3 $\alpha$ complex, but overexpression and depletion of PP4IP impair DNA DSB repairs and cell survival, suggesting that timely regulation of PP4 is essential for DNA repair and cell survival (49). And TIPRL downregulates PP4 activity by inhibiting the interaction between PP4C and PP4R2 by interception of PP4C from PP4C-PP4R2 complex (48). The fact that no synthetic inhibitor has been discovered makes it attractive to study the development of specific inhibitor to PP4. The PP4-specific inhibitor that has no impact on other phosphatases will be applied as beneficial material for treating PP4-overexpressing cancers and investigating PP4 functions in more detail.

Similar to mammalian cells, PP4 is also regarded as an essential element in yeast DDR. PPH3 prevents hyperphosphorylation of Rad53 (checkpoint kinase 2, CHK2, yeast homolog), thus affecting DNA end resection at DSBs (14), and also promotes telomere healing at accidental breaks by opposing Cdc13 phosphorylation, even though it is unknown whether PPH3 directly dephosphorylates Cdc13 (50). Besides, PPH3 depletion alleviates DNA damage accumulation and rescues the short lifespan in maf1 $\Delta$ cells (51). Maf1, a repressor of RNA polymerase III transcription, was reported to be dephosphorylated by $\mathrm{PPH} 3$ during unfavorable conditions, such as nutrient deprivation (52).

Not always, but in many cases, protein functions in yeast homolog are reflected in the human system. Thus, it is quite convincing that human PP4 regulates CHK2-mediated activity and telomere-related events. Actually, the phosphorylation of KAP-1 by CHK2 is regulated by PP4 and hyperphosphorylation of 53BP1 in mitosis induced by PP4 inactivation prevents telomere fusion $(6,53)$.

\section{Immunity}

Immune cell development and activation are regulated by extremely sophisticated pathways, and dysfunction of these processes leads to many hazardous immune disorders. To prevent such catastrophic incidents, PP4 participates in various immune-related processes, such as immune cell lineage development, cytokine secretion, and immune cell receptor signaling.

Pp4 gene knockout in the T-cell lineage causes aberrant thymocyte development, including $\mathrm{T}$ cell arrest at the doublenegative 3 stage $\left(\mathrm{CD} 4{ }^{-} \mathrm{CD} 8{ }^{-} \mathrm{CD} 25^{+} \mathrm{CD} 44^{-}\right)$, abnormal thymocyte maturation, and lower efficacy of positive selection with impaired PLC $\gamma 1$ (phospholipase C- $\gamma 1$ )-ERK (extracellular signal-regulated kinase) activation (54). Also, PP4 deficiency induces partial $\alpha \beta$ T lymphopenia and T cell hypo-proliferation, as well as significant reductions in the numbers of thymic and peripheral Treg cells (regulatory $\mathrm{T}$ cells), thus inducing defective adaptive immunity (55). These aberrations are associated with decreased IL-10, CTLA-4 (cytotoxic T-lymphocyteassociated protein 4), GITR (glucocorticoid-induced TNFRrelated protein), and CD103 expression, elevated transcriptional expression of CDK inhibitors including p15, p16, and p21, and enhanced AMPK (AMP kinase) activation $(55,56)$. Also, mice with $\mathrm{T}$ cell-specific ablation of the $p p 4 c$ gene develop spontaneous rectal prolapse and colitis with symptom similar to human Crohn's disease (55). Besides, PP4 is related to signaling pathways in T cells. HPK1 (hematopoietic progenitor kinase 1), a member of mammalian Ste20-like protein kinases, has been implicated in many cellular signaling pathways including TCR and BCR ( $T$ cell and B cell receptor, respectively) signaling. TCR stimulation promotes the interaction between PP4 and HPK1, and PP4 induces TCR-mediated activation of HPK1 in Jurkat T cells (57). Also, the activation of JNK and p38, but not ERKs, is a target for the PP4 in the Jurkat cell line (58).

PP4 is equally essential for B-cell lineage development. Ablation of PP4 in B-cell lineage leads to reduction in pre-B cell numbers, an absence in immature $B$ cells, and a complete loss of mature B cells (59). In the PP4-knockout B cells, immunoglobulin (Ig) class switch recombination is impaired and the basal levels of serum immunoglobulins of all isotypes are reduced (59-61). However, beyond the cell proliferation phase, the conditional deletion of PP4 completely restores normal IgG1 production in B cells of immunized mice (61). The pp4 gene-ablated mice fail to form germinal centers in the spleen and the draining mediastinal lymph nodes, and do not efficiently mount antigen-specific humoral response, associated with lower activation of ERK and JNK, and $I \kappa B \alpha$ (inhibitor of $\kappa B \alpha$ ) degradation, both of which are mediated by CD40 (60).

In addition to roles in T and B cells, PP4 is an essential component in other immune cells including macrophage. Type I IFN production is indispensable for antiviral innate immune response, and TBK1 (TANK-binding kinase 1) plays crucial roles in type I IFN production. PP4 suppresses production of type I IFN and IFN-stimulated genes by dephosphorylating and inhibiting TBK1 (62).

Similar to the conflicting role in genomic stability, the overexpression and depletion of PP4 cause apoptosis in T cells, meaning that PP4 can be proapoptotic or antiapoptotic gene $(54,63,64)$. Interestingly, the knockout of PP4 in rodents causes embryonic lethality (54), suggesting that tight regulation or adequate expression of PP4 is pivotal in immune system development, at least in T cell lineage. 


\section{Glucose homeostasis}

The dysfunction of glucose homeostasis leads to critical metabolic disorders, such as diabetes and obesity. Insulin resistance is one of the main causes contributing to impaired glucose dysregulation (65). Recently, accumulating data indicate that PP4 is related to insulin resistance and glucose metabolism.

In type 2 diabetic $\mathrm{db} / \mathrm{db}$ mice or insulin-resistant mice treated with TNF- $\alpha$ (tumor necrosis factor $\alpha$ ), the expression of PP4C and PP4R1 in protein level and PP4R3 $\alpha / \beta$ in mRNA level is increased, and downregulation of PP4 alleviates the insulin resistance (66-69), though the alteration of PP4R2 expression level remains elusive. It was reported that TNF- $\alpha$ induces the phosphorylation and activation of PP4C, subsequently leading to the activation of JNK (70). However, it seems that PP4 may regulate JNK function in an indirect manner, since PP4 does not physically interact with JNK (70). Also, upon the activation of JNK, the interaction of IRS-1 (insulin receptor substrate 1) with PP4 causes the decreased expression of IRS-1 and increased phosphorylation of IRS-1 (68). Additionally, TNF- $\alpha$ downregulates IRS-4 expression, which depends on the phosphatase activity of PP4. But, it is unknown whether PP4 dephosphorylates IRS-4 directly (71).

ACC1 (acetyl-CoA carboxylase 1) is associated with hepatic lipogenesis, and its phosphorylation by AMPK blocks lipid synthesis and is reversed by PP4 (66). Consistently, PP4 dephosphorylates AMPK in $\mathrm{Ca}^{2+}$ dependent manner, thus blocking lipid consumption (72). Furthermore, PP4 is involved in gluconeogenesis. Overexpression of $\mathrm{PP} 4 \mathrm{R} 3 \alpha / \beta$ induces dephosphorylation of CRTC2 (CAMP-response element binding protein-regulated transcriptional coactivator 2) and promotes transcription of gluconeogenesis-related genes (69). It remains to be seen whether PP4 directly dephosphorylates CRTC2. Also, as another role in glucose signaling, PPH3 and PSY2 (PP4C and PP4R3 yeast homolog, respectively) dephosphorylate Mth1 (MutT homolog 1) in glucose withdrawal condition, causing the binding of Rgt1 (restores glucose transport protein 1) to the promoters of glucose transporter (HXT) genes and represses their expression (73).

\section{Neuronal development}

SMA (Spinal muscular atrophy) is an autosomal recessive neurodegenerative disease characterized by progressive loss of motor neurons from the anterior horn of the spinal cord, resulting in paralysis and severe muscular atrophy (74). SMA mainly arises from deletions or mutations in SMN1 (Survival of Motor Neuron 1) gene and its abnormal high expression. SMN complexes participate in spliceosome assembly and regeneration of spliceosomal components. PP4R2 interacts with Gemin3 and Gemin4, identified as components of the SMN protein complex. Also, PP4R2 promotes the temporal localization of newly formed snRNPs and modulates the differentiation and survival of neuronal cells $(74,75)$.

Hedgehog signaling is related to the development in vertebrates and invertebrates, and Smo (the seven-transmembrane protein smoothened) is an essential factor in Hedgehog signaling (76). In Drosophila, PP4 dephosphorylates Smo and alleviates its signaling activity (77). Wnt signaling regulates growth and proliferation, cell-fate differentiation, stem cell renewal and homeostasis (78). In embryonic stem cells, PP4R3 $\alpha / \beta$ suppresses the transcription of Wnt target gene brachyury through recruitment of HDAC1 (histone deacetylase 1) to its promoter to maintain pluripotency (79). However, in Drosophila development, PP4 promotes the Notch signaling to induce expression of wg (wingless), and Ryk (Receptor-like tyrosine kinase; a Wnt receptor) intracellular domain, in complex with $\mathrm{PP} 4 \mathrm{R} 3 \alpha / \beta$ that regulates the gene transcription for neuronal differentiation $(78,80)$. Additionally, PP4 promotes the axonal regeneration after only peripheral injury, but not spinal cord injury, by dephosphorylating and inactivating HDAC3 (81). Similarly, HDAC3 activity is inhibited by PP4C/PP4R1 complex in HeLa cell, a cervical cancer cell line (82). Also, PP4R3 $\alpha / \beta$ induces Mbd3 (methyl-CpG-binding domain 3) degradation and blocks recruitment of the repressive Mbd3/NuRD (nucleosome remodeling and deacetylase) complex to the neurogenesis-related gene loci (83). Interestingly, the clearance of severed or degenerating axons also requires PP4 in drosophila, bridging the glial receptor Draper to cytoskeletal remodeling through the SOS GEF (guanine nucleotide exchange factor) complex and the GTPase Rac1 to ensure glial infiltration of injury sites and clearance of degenerating neuronal materials (84).

Drosophila NBs (neuroblasts), stem cell-like neural progenitors, undergo repeated asymmetric divisions to self-renew and generate neurons and/or glia. During each round of division, the cell fate determinants, such as Pros (Prospero) and Brat (Brain Tumor), are asymmetrically localized and preferentially segregated into the GMCs (ganglion mother cells). Asymmetric localization of Pros and Brat is mediated through direct interactions with Mira (Miranda) (85). Pp4-19C or PPP4R2r (PP4C or PP4R2 Drosophila homologs) facilitate localization of Mira and PP4R3/FIfl interacts directly with Mira $(85,86)$. Also, PP4 complex associates with PTPA (phosphotyrosyl phosphatase activator), thus inducing dephosphorylation of Mira and directing cortical localization of Mira (86). In developing mammalian brains, neural progenitors expand by symmetric division before switching to an asymmetric division mode to generate neurons (87). PP4 facilitates mitotic spindles orientation in parallel to the neuroepithelial surface through NDEL1 dephosphorylation, thus preventing premature neurogenesis (88). Conversely, it was reported that PP4/PP4R3 $\alpha$ complex promotes neuronal differentiation and suppresses the proliferative capacity of neural progenitor cells by suppressing Par3, a negative regulator of neuronal differentiation (89).

\section{Plant and others}

PP4 functions also have been elucidated in other various biological fields, though requiring more information in future. First, Arabidopsis thaliana as a model organism in plant biology 
has two PP4 catalytic subunit genes, PPX1 and PPX2. PP4R3A, with these catalytic subunits, assembles a functional PP4 complex to target Hyponastic Leaves 1, HYL1 (90). HYL1 has a role in promoting miRNA biogenesis, antagonized and degraded by the MAPK pathway. PP4 complexes stabilize and also dephosphorylate HYL1 to promote miRNA biogenesis, inhibiting MAPK signal cascade. Also, PP4R3A interacts with RNA polymerase II and recruits it to the promoters of miRNAencoding (MIR) genes (91). Additionally, it was shown that PP4R3A gene is related to plant growth, root morphology, and genomic integrity in Arabidopsis thaliana (92).

PP4 can activate or inhibit NF- $\kappa B$ signaling. Signaling to NF$\kappa \mathrm{B}$ is crucial for $\mathrm{T}$ cell activation, differentiation, and proliferation as it regulates a wide variety of target genes such as different cytokines (e.g., IL-2, IFN- $\gamma$, and TNF- $\alpha$ ), chemokines (e.g., IL-8), and anti-apoptotic molecules (e.g., Bcl-2 and c-IAPs; cellular inhibitor of apoptosis protein 1) (93). PP4C-PP4R1 complex functions as the negative regulator between $I \kappa B$ and IKK (IKB kinase) complex. Inactivation of IKK by PP4C/PP4R1mediated dephosphorylation keeps IKB dephosphorylated and thereby, prevents the activation of NF-אB (93). PP4 also negatively regulates LPS-induced and TRAF6 (TNF receptor-associated factor 6)-mediated NF-KB activation by inhibiting the ubiquitination of TRAF6 (94). Although PP4 physically interacts with TRAF6 and is recruited to TLR4 (toll-like receptor 4) complex upon LPS (lipopolysaccharide) stimulation, whether PP4 regulates TRAF6 by direct dephosphorylation remains to be elucidated.

In non-immune cells, PP4 stimulates c-Rel-mediated DNA binding and NF- $\mathrm{KB}$-mediated transcription, and decreases threonine phosphorylation on p65 subunit of NF-kB for its activation $(95,96)$. Conversely, PP4 can also inactivate NF-KB activity by dephosphorylation of TRAF2, inhibition of TRAF6 polyubiquitination, and interaction with tAg (small $\mathrm{T}$ antigen) of MCPyV (merkel cell polyomavirus) and NEMO (NF-KB essential modulator) adaptor protein $(97,98)$.

In Neurospora, PP4 dephosphorylates and activates WHITE COLLAR complex (WCC), phosphorylated by FREQUENCYmediated manner to close circadian negative feedback loop (99). Additionally, PP4 inhibits thiazide-sensitive $\mathrm{Na}^{+}-\mathrm{Cl}^{-}$ cotransporter (NCC) in Xenopus oocytes, but it is unknown that PP4 directly dephosphorylates NCC, although its phosphatase activity is required for NCC inhibition (100). Furthermore, PP4 is expressed in the distal convoluted tubule of mouse kidney (100). These data suggest the possible role of PP4 in the regulation of the electrolyte transporter.

\section{CONCLUSION}

Emerging evidence clearly suggests that PP4 plays multifaceted roles which control a variety of cellular phenomena. In the light of the significant impact of PP4 in cells, it is obvious that a more intensive study should be pursued to achieve better understanding of the working mechanisms in organism, as well as to clarify conflicting results observed in the different experimental systems. In that scenario, PP4 could be harnessed for the development of therapeutic materials. For example, since PP4C is considered an oncoprotein frequently overexpressed or irrelevantly regulated in a number of tumor types, discovering molecules as an inhibitor suppressing or blocking the gene expression or inhibiting directly enzymatic functions of PP4 is sufficiently persuasive and promising. From another perspective, the synthetic inhibitor, specific to PP4C, can be developed applying an entire or functional region of endogenous protein inhibitors identified. Inhibitors has been used in the studies on PP4 also inhibits other phosphatases meaning that there is no PP4-specific inhibitor developed. The PP4 inhibitor to be produced should be valuable for investigating PP4specific functions and targeting tumors particularly with the high level of PP4C expression in the long term.

\section{ACKNOWLEDGEMENTS}

This work was supported by the Basic Science Research Program through the National Research Foundation of Korea (NRF) funded by the Ministry of Science, ICT and Future Planning (grant number: NRF-2017R1A2B4007852).

\section{CONFLICTS OF INTEREST}

The authors have no conflicting interests.

\section{REFERENCES}

1. Brautigan DL (2013) Protein Ser/Thr phosphatases-the ugly ducklings of cell signalling. FEBS J 280, 324-345

2. Lillo C, Kataya AR, Heidari B et al (2014) Protein phosphatases PP2A, PP4 and PP6: mediators and regulators in development and responses to environmental cues. Plant Cell Environ 37, 2631-2648

3. Lee DH and Chowdhury D (2011) What goes on must come off: phosphatases gate-crash the DNA damage response. Trends Biochem Sci 36, 569-577

4. Brewis ND, Street AJ, Prescott AR and Cohen PT (1993) PPX, a novel protein serine/threonine phosphatase localized to centrosomes. EMBO J 12, 987-996

5. Lee $\mathrm{DH}$, Pan $\mathrm{Y}$, Kanner $\mathrm{S}$, Sung $\mathrm{P}$, Borowiec JA and Chowdhury D (2010) A PP4 phosphatase complex dephosphorylates RPA2 to facilitate DNA repair via homologous recombination. Nat Struct Mol Biol 17, 365-372

6. Lee DH, Goodarzi AA, Adelmant GO et al (2012) Phosphoproteomic analysis reveals that PP4 dephosphorylates $\mathrm{KAP}-1$ impacting the DNA damage response. EMBO J 31, 2403-2415

7. Lee J, Adelmant G, Marto JA and Lee DH (2015) Dephosphorylation of DBC1 by Protein Phosphatase 4 Is Important for p53-Mediated Cellular Functions. Mol Cells 38, 697-704

8. Lee DH, Acharya SS, Kwon M et al (2014) Dephosphorylation enables the recruitment of 53BP1 to double- 
strand DNA breaks. Mol Cell 54, 512-525

9. Chowdhury D, Xu X, Zhong X et al (2008) A PP4-phosphatase complex dephosphorylates gamma-H2AX generated during DNA replication. Mol Cell 31, 33-46

10. Chen GI, Tisayakorn S, Jorgensen C, D'Ambrosio LM, Goudreault M and Gingras AC (2008) PP4R4/KIAA1622 forms a novel stable cytosolic complex with phosphoprotein phosphatase 4. J Biol Chem 283, 29273-29284

11. Perez-Callejon E, Casamayor A, Pujol G, Clua E, Ferrer A and Arino J (1993) Identification and molecular cloning of two homologues of protein phosphatase $X$ from Arabidopsis thaliana. Plant Mol Biol 23, 1177-1185

12. Wu HI, Brown JA, Dorie MJ, Lazzeroni L and Brown JM (2004) Genome-wide identification of genes conferring resistance to the anticancer agents cisplatin, oxaliplatin, and mitomycin C. Cancer Res 64, 3940-3948

13. Gingras AC, Caballero M, Zarske M et al (2005) A novel, evolutionarily conserved protein phosphatase complex involved in cisplatin sensitivity. Mol Cell Proteomics 4, $1725-1740$

14. Villoria MT, Gutierrez-Escribano P, Alonso-Rodriguez E et al (2019) PP4 phosphatase cooperates in recombinational DNA repair by enhancing double-strand break end resection. Nucleic Acids Res 47, 10706-10727

15. Wang B, Zhao A, Sun L et al (2008) Protein phosphatase PP4 is overexpressed in human breast and lung tumors. Cell Res 18, 974-977

16. Weng S, Wang $\mathrm{H}$, Chen $\mathrm{W}$ et al (2012) Overexpression of protein phosphatase 4 correlates with poor prognosis in patients with stage II pancreatic ductal adenocarcinoma. Cancer Epidemiol Biomarkers Prev 21, 1336-1343

17. Li X, Liang L, Huang L, Ma X, Li D and Cai S (2015) High expression of protein phosphatase 4 is associated with the aggressive malignant behavior of colorectal carcinoma. Mol Cancer 14, 95

18. Li M, Li X, Xu S et al (2016) Protein phosphatase 4 catalytic subunit is overexpressed in glioma and promotes glioma cell proliferation and invasion. Tumour Biol 37, 11893-11901

19. Inostroza J, Saenz L, Calaf G, Cabello G and Parra E (2005) Role of the phosphatase PP4 in the activation of JNK-1 in prostate carcinoma cell lines PC-3 and LNCaP resulting in increased AP-1 and EGR-1 activity. Biol Res 38, 163-178

20. Herzig JK, Bullinger L, Tasdogan A et al (2017) Protein phosphatase 4 regulatory subunit 2 (PPP4R2) is recurrently deleted in acute myeloid leukemia and required for efficient DNA double strand break repair. Oncotarget 8, 95038-95053

21. Wang $\mathrm{H}$, Chen $\mathrm{Y}$, Lin $\mathrm{P}$ et al (2014) The CUL7/F-box and WD repeat domain containing 8 (CUL7/Fbxw8) ubiquitin ligase promotes degradation of hematopoietic progenitor kinase 1. J Biol Chem 289, 4009-4017

22. Woo SM, Min KJ and Kwon TK (2012) Calyculin A causes sensitization to tumor necrosis factor-related apoptosis-inducing ligand (TRAIL)-induced apoptosis by ROS-mediated down-regulation of cellular FLICE-inhibiting protein (c-FLIP) and by enhancing death receptor 4 mRNA stabilization. Apoptosis 17, 1223-1234

23. Wu G, Ma Z, Qian J and Liu B (2015) PP4R1 accelerates cell growth and proliferation in HepG2 hepatocellular carcinoma. Onco Targets Ther 8, 2067-2074

24. Mourtada-Maarabouni M and Williams GT (2008) Protein phosphatase 4 regulates apoptosis, proliferation and mutation rate of human cells. Biochim Biophys Acta 1783, 1490-1502

25. Mohammed HN, Pickard MR and Mourtada-Maarabouni $M$ (2016) The protein phosphatase 4-PEA15 axis regulates the survival of breast cancer cells. Cell Signal $28,1389-1400$

26. Helps NR, Brewis ND, Lineruth K, Davis T, Kaiser K and Cohen PT (1998) Protein phosphatase 4 is an essential enzyme required for organisation of microtubules at centrosomes in Drosophila embryos. J Cell Sci 111 ( Pt 10), 1331-1340

27. Sumiyoshi E, Sugimoto A and Yamamoto M (2002) Protein phosphatase 4 is required for centrosome maturation in mitosis and sperm meiosis in C. elegans. J Cell Sci 115, 1403-1410

28. Han X, Gomes JE, Birmingham CL, Pintard L, Sugimoto $A$ and Mains PE (2009) The role of protein phosphatase 4 in regulating microtubule severing in the Caenorhabditis elegans embryo. Genetics 181, 933-943

29. Toyo-oka K, Mori D, Yano Y et al (2008) Protein phosphatase 4 catalytic subunit regulates Cdk1 activity and microtubule organization via NDEL1 dephosphorylation. J Cell Biol 180, 1133-1147

30. Voss M, Campbell K, Saranzewa N et al (2013) Protein phosphatase 4 is phosphorylated and inactivated by $\mathrm{Cdk}$ in response to spindle toxins and interacts with gammatubulin. Cell Cycle 12, 2876-2887

31. Martin-Granados C, Philp A, Oxenham SK, Prescott AR and Cohen PT (2008) Depletion of protein phosphatase 4 in human cells reveals essential roles in centrosome maturation, cell migration and the regulation of Rho GTPases. Int J Biochem Cell Biol 40, 2315-2332

32. Lipinszki Z, Lefevre S, Savoian MS, Singleton MR, Glover DM and Przewloka MR (2015) Centromeric binding and activity of Protein Phosphatase 4. Nat Commun 6, 5894

33. Sato-Carlton A, Li X, Crawley O et al (2014) Protein phosphatase 4 promotes chromosome pairing and synapsis, and contributes to maintaining crossover competence with increasing age. PLoS Genet 10, e1004638

34. Falk JE, Chan AC, Hoffmann E and Hochwagen A (2010) A Mec1- and PP4-dependent checkpoint couples centromere pairing to meiotic recombination. Dev Cell 19 599-611

35. Birot A, Eguienta K, Vazquez S et al (2017) A second Wpl1 anti-cohesion pathway requires dephosphorylation of fission yeast kleisin Rad21 by PP4. EMBO J 36, 1364-1378

36. Ueki Y, Kruse T, Weisser MB et al (2019) A Consensus Binding Motif for the PP4 Protein Phosphatase. Mol Cell 76, 953-964 e6

37. Jablonowski CM, Cussiol JR, Oberly S et al (2015) Termination of Replication Stress Signaling via Concerted Action of the Slx4 Scaffold and the PP4 Phosphatase. Genetics 201, 937-949

38. Hustedt N, Seeber A, Sack R et al (2015) Yeast PP4 interacts with ATR homolog Ddc2-Mec1 and regulates 
checkpoint signaling. Mol Cell 57, 273-289

39. Zhuang X, Semenova E, Maric D and Craigie R (2014) Dephosphorylation of barrier-to-autointegration factor by protein phosphatase 4 and its role in cell mitosis. J Biol Chem 289, 1119-1127

40. Huang X, Liu J, Shen T et al (2016) Protein phosphatase 4 plays dual roles during cell proliferation. Cell Prolif 49, 219-235

41. Shaltiel IA, Aprelia M, Saurin AT et al (2014) Distinct phosphatases antagonize the p53 response in different phases of the cell cycle. Proc Natl Acad Sci U S A 111, 7313-7318

42. Nakada S, Chen GI, Gingras AC and Durocher D (2008) PP4 is a gamma $\mathrm{H} 2 \mathrm{AX}$ phosphatase required for recovery from the DNA damage checkpoint. EMBO Rep 9, 10191026

43. Liu J, Xu L, Zhong J, Liao J, Li J and Xu X (2012) Protein phosphatase PP4 is involved in NHEJ-mediated repair of DNA double-strand breaks. Cell Cycle 11, 2643-2649

44. Lee J and Lee DH (2014) Leucine methylation of protein phosphatase PP4C at C-terminal is critical for its cellular functions. Biochem Biophys Res Commun 452, 42-47

45. Hwang J, Lee JA and Pallas DC (2016) Leucine Carboxyl Methyltransferase 1 (LCMT-1) Methylates Protein Phosphatase 4 (PP4) and Protein Phosphatase 6 (PP6) and Differentially Regulates the Stable Formation of Different PP4 Holoenzymes. J Biol Chem 291, 21008-21019

46. Zheng XF, Acharya SS, Choe KN et al (2019) A mitotic CDK5-PP4 phospho-signaling cascade primes 53BP1 for DNA repair in G1. Nat Commun 10, 4252

47. Han S, Park J and Lee DH (2015) Protein DHX38 is a novel inhibitor of protein phosphatase 4. Animal Cells Syst 19, 236-244

48. Rosales KR, Reid MA, Yang Y et al (2015) TIPRL Inhibits Protein Phosphatase 4 Activity and Promotes H2AX Phosphorylation in the DNA Damage Response. PLoS One 10, e0145938

49. Park J, Lee J and Lee DH (2019) Identification of Protein Phosphatase 4 Inhibitory Protein That Plays an Indispensable Role in DNA Damage Response. Mol Cells 42, 546-556

50. Zhang W and Durocher D (2010) De novo telomere formation is suppressed by the Mec1-dependent inhibition of Cdc13 accumulation at DNA breaks. Genes Dev 24, 502-515

51. Shetty M, Noguchi C, Wilson S et al (2019) Maf1-dependent transcriptional regulation of tRNAs prevents genomic instability and is associated with extended lifespan. Aging Cell, e13068

52. Oler AJ and Cairns BR (2012) PP4 dephosphorylates Maf1 to couple multiple stress conditions to RNA polymerase III repression. EMBO J 31, 1440-1452

53. Orthwein A, Fradet-Turcotte A, Noordermeer SM et al (2014) Mitosis inhibits DNA double-strand break repair to guard against telomere fusions. Science 344, 189-193

54. Shui JW, Hu MC and Tan TH (2007) Conditional knockout mice reveal an essential role of protein phosphatase 4 in thymocyte development and pre-T-cell receptor signaling. Mol Cell Biol 27, 79-91

55. Liao FH, Shui JW, Hsing EW et al (2014) Protein phosphatase 4 is an essential positive regulator for Treg development, function, and protective gut immunity. Cell Biosci 4, 25

56. Liao FH, Hsiao WY, Lin YC, Chan YC and Huang CY (2016) T cell proliferation and adaptive immune responses are critically regulated by protein phosphatase 4 . Cell Cycle 15, 1073-1083

57. Zhou G, Boomer JS and Tan TH (2004) Protein phosphatase 4 is a positive regulator of hematopoietic progenitor kinase 1. J Biol Chem 279, 49551-49561

58. Parra E (2012) Activation of MAP kinase family members triggered by TPA or ionomycin occurs via the protein phosphatase 4 pathway in Jurkat leukemia T cells. Mol Med Rep 5, 773-778

59. Su YW, Chen YP, Chen MY, Reth M and Tan TH (2013) The serine/threonine phosphatase PP4 is required for pro-B cell development through its promotion of immunoglobulin VDJ recombination. PLoS One 8, e68804

60. Chen MY, Chen YP, Wu MS et al (2014) PP4 is essential for germinal center formation and class switch recombination in mice. PLoS One 9, e107505

61. Chen MY, Hsu WC, Hsu SC et al (2019) PP4 deficiency leads to DNA replication stress that impairs immunoglobulin class switch efficiency. Cell Death Differ 26, 1221-1234

62. Zhan Z, Cao H, Xie X et al (2015) Phosphatase PP4 Negatively Regulates Type I IFN Production and Antiviral Innate Immunity by Dephosphorylating and Deactivating TBK1. J Immunol 195, 3849-3857

63. Mourtada-Maarabouni M, Kirkham L, Jenkins B et al (2003) Functional expression cloning reveals proapoptotic role for protein phosphatase 4. Cell Death Differ 10, 1016-1024

64. Mourtada-Maarabouni M and Williams GT (2009) Protein phosphatase 4 regulates apoptosis in leukemic and primary human T-cells. Leuk Res 33, 1539-1551

65. Hall H, Perelman D, Breschi A et al (2018) Glucotypes reveal new patterns of glucose dysregulation. PLoS Biol $16, \mathrm{e} 2005143$

66. Meng X, Li M, Guo J et al (2014) Protein phosphatase 4 promotes hepatic lipogenesis through dephosphorylating acetyl-CoA carboxylase 1 on serine 79. Mol Med Rep 10, 1959-1963

67. Dou L, Wang S, Sun L et al (2017) Mir-338-3p Mediates Tnf-A-Induced Hepatic Insulin Resistance by Targeting PP4r1 to Regulate PP4 Expression. Cell Physiol Biochem 41, 2419-2431

68. Zhao H, Huang X, Jiao J et al (2015) Protein phosphatase 4 (PP4) functions as a critical regulator in tumor necrosis factor (TNF)-alpha-induced hepatic insulin resistance. Sci Rep 5, 18093

69. Yoon YS, Lee MW, Ryu D et al (2010) Suppressor of MEK null (SMEK)/protein phosphatase 4 catalytic subunit (PP4C) is a key regulator of hepatic gluconeogenesis. Proc Natl Acad Sci U S A 107, 17704-17709

70. Zhou G, Mihindukulasuriya KA, MacCorkle-Chosnek RA et al (2002) Protein phosphatase 4 is involved in tumor necrosis factor-alpha-induced activation of c-Jun $\mathrm{N}$-terminal kinase. J Biol Chem 277, 6391-6398

71. Mihindukulasuriya KA, Zhou G, Qin J and Tan TH 
(2004) Protein phosphatase 4 interacts with and downregulates insulin receptor substrate 4 following tumor necrosis factor-alpha stimulation. J Biol Chem 279, 46588-46594

72. Tomar D, Jana F, Dong Z et al (2019) Blockade of MCUMediated $\mathrm{Ca}(2+)$ Uptake Perturbs Lipid Metabolism via PP4-Dependent AMPK Dephosphorylation. Cell Rep 26, 3709-3725 e3707

73. Ma H, Han BK, Guaderrama M et al (2014) Psy2 targets the PP4 family phosphatase Pph3 to dephosphorylate Mth1 and repress glucose transporter gene expression. Mol Cell Biol 34, 452-463

74. Carnegie GK, Sleeman JE, Morrice N et al (2003) Protein phosphatase 4 interacts with the Survival of Motor Neurons complex and enhances the temporal localisation of snRNPs. J Cell Sci 116, 1905-1913

75. Bosio Y, Berto G, Camera P et al (2012) PPP4R2 regulates neuronal cell differentiation and survival, functionally cooperating with SMN. Eur J Cell Biol 91, 662-674

76. Gupta S, Takebe N and Lorusso P (2010) Targeting the Hedgehog pathway in cancer. Ther Adv Med Oncol 2, 237-250

77. Jia H, Liu Y, Yan W and Jia J (2009) PP4 and PP2A regulate Hedgehog signaling by controlling $\mathrm{Smo}$ and $\mathrm{Ci}$ phosphorylation. Development 136, 307-316

78. Hall ET, Pradhan-Sundd T, Samnani F and Verheyen EM (2017) The protein phosphatase 4 complex promotes the Notch pathway and wingless transcription. Biol Open 6, 1165-1173

79. Lyu J, Jho EH and Lu W (2011) Smek promotes histone deacetylation to suppress transcription of Wnt target gene brachyury in pluripotent embryonic stem cells. Cell Res 21, 911-921

80. Chang WH, Choi SH, Moon BS et al (2017) Smek1/2 is a nuclear chaperone and cofactor for cleaved Wnt receptor Ryk, regulating cortical neurogenesis. Proc Natl Acad Sci U S A 114, E10717-E10725

81. Hervera A, Zhou L, Palmisano I et al (2019) PP4dependent HDAC3 dephosphorylation discriminates between axonal regeneration and regenerative failure. $\mathrm{EMBO}$ J 38, e101032

82. Zhang X, Ozawa $\mathrm{Y}$, Lee $\mathrm{H}$ et al (2005) Histone deacetylase 3 (HDAC3) activity is regulated by interaction with protein serine/threonine phosphatase 4. Genes Dev 19, 827-839

83. Moon BS, Yun HM, Chang WH et al (2017) Smek promotes corticogenesis through regulating Mbd3's stability and $\mathrm{Mbd} 3 / \mathrm{NuRD}$ complex recruitment to genes associated with neurogenesis. PLoS Biol 15, e2001220

84. Winfree LM, Speese SD and Logan MA (2017) Protein phosphatase 4 coordinates glial membrane recruitment and phagocytic clearance of degenerating axons in Drosophila. Cell Death Dis 8, e2623

85. Sousa-Nunes R, Chia W and Somers WG (2009) Protein phosphatase 4 mediates localization of the Miranda complex during Drosophila neuroblast asymmetric divisions. Genes Dev 23, 359-372

86. Zhang F, Huang ZX, Bao H et al (2016) Phosphotyrosyl phosphatase activator facilitates localization of Miranda through dephosphorylation in dividing neuroblasts. Development 143, 35-44

87. Gotz $M$ and Huttner WB (2005) The cell biology of neurogenesis. Nat Rev Mol Cell Biol 6, 777-788

88. Xie Y, Juschke $C$, Esk $C$, Hirotsune $S$ and Knoblich JA (2013) The phosphatase PP4c controls spindle orientation to maintain proliferative symmetric divisions in the developing neocortex. Neuron 79, 254-265

89. Lyu J, Kim HR, Yamamoto V et al (2013) Protein phosphatase 4 and Smek complex negatively regulate Par3 and promote neuronal differentiation of neural stem/ progenitor cells. Cell Rep 5, 593-600

90. Su C, Li Z, Cheng J et al (2017) The Protein Phosphatase 4 and SMEK1 Complex Dephosphorylates HYL1 to Promote miRNA Biogenesis by Antagonizing the MAPK Cascade in Arabidopsis. Dev Cell 41, 527-539 e525

91. Wang S, Quan L, Li S et al (2019) The PROTEIN PHOSPHATASE4 Complex Promotes Transcription and Processing of Primary microRNAs in Arabidopsis. Plant Cell 31, 486-501

92. Kataya ARA, Creighton MT, Napitupulu TP et al (2017) PLATINUM SENSITIVE 2 LIKE impacts growth, root morphology, seed set, and stress responses. PLoS One 12, e0180478

93. Brechmann M, Mock T, Nickles D et al (2012) A PP4 holoenzyme balances physiological and oncogenic nuclear factor-kappa B signaling in T lymphocytes. Immunity $37,697-708$

94. Chen L, Dong W, Zou T et al (2008) Protein phosphatase 4 negatively regulates LPS cascade by inhibiting ubiquitination of TRAF6. FEBS Lett 582, 2843-2849

95. Hu MC, Tang-Oxley Q, Qiu WR et al (1998) Protein phosphatase X interacts with c-Rel and stimulates c-Rel/ nuclear factor kappaB activity. J Biol Chem 273, 3356133565

96. Yeh PY, Yeh KH, Chuang SE, Song YC and Cheng AL (2004) Suppression of MEK/ERK signaling pathway enhances cisplatin-induced NF-kappaB activation by protein phosphatase 4-mediated NF-kappaB p65 Thr dephosphorylation. J Biol Chem 279, 26143-26148

97. Hadweh $P$, Habelhah $H$, Kieff E, Mosialos $G$ and Hatzivassiliou E (2014) The PP4R1 subunit of protein phosphatase PP4 targets TRAF2 and TRAF6 to mediate inhibition of NF-kappaB activation. Cell Signal 26, 27302737

98. Abdul-Sada H, Muller M, Mehta R et al (2017) The PP4R1 sub-unit of protein phosphatase PP4 is essential for inhibition of NF-kappaB by merkel polyomavirus small tumour antigen. Oncotarget 8, 25418-25432

99. Cha J, Chang SS, Huang G, Cheng P and Liu Y (2008) Control of WHITE COLLAR localization by phosphorylation is a critical step in the circadian negative feedback process. EMBO J 27, 3246-3255

100. Glover M, Mercier Zuber A, Figg N and O'Shaughnessy KM (2010) The activity of the thiazide-sensitive $\mathrm{Na}(+)-\mathrm{Cl}(-)$ cotransporter is regulated by protein phosphatase PP4. Can J Physiol Pharmacol 88, 986-995 